Supporting Info.

\title{
Tandem Nazarov Cyclization-Michael Addition Sequence Catalyzed by an Ir (III) Complex
}

Mesfin Janka, Wei He, Inga Haedicke, Frank R. Fronczek, Alison J. Frontier* and Richard Eisenberg* Department of Chemistry, University of Rochester, Rochester, NY 14627

\section{Supporting Information}

Page \#

I. Experimental details
a) General procedures and materials
b) Syntheses of Catalyst, Nazarov Substrates and Nitrostyrene

II. General procedure and characterization data for $[\operatorname{IrMe}(\mathrm{CO})(\mathrm{dppe})(\mathrm{DIB})]\left(\mathrm{BAr}_{4}\right)_{2}$ catalyzed tandem Nazarov cyclization-Michael addition
a) General procedure
S-3
b) Characterization of tandem products $\mathbf{4 a - 4 i}$
S-3 to $\mathrm{S}-8$
c) ${ }^{1} \mathrm{H}$ NMR spectrum of tandem product $4 \mathbf{a}$
S-9
d) ${ }^{13} \mathrm{C}\left\{{ }^{1} \mathrm{H}\right\},{ }^{13} \mathrm{C} / \mathrm{DEPT} 135$ and ${ }^{13} \mathrm{C} / \mathrm{DEPT} 90 \mathrm{NMR}$ spectra of $4 \mathbf{a}$
S-10
e) ${ }^{1} \mathrm{H}$ NMR spectrum of tandem product $\mathbf{4 b}$
f) ${ }^{13} \mathrm{C}\left\{{ }^{1} \mathrm{H}\right\},{ }^{13} \mathrm{C} / \mathrm{DEPT} 135$ and ${ }^{13} \mathrm{C} / \mathrm{DEPT} 90 \mathrm{NMR}$ spectra of $\mathbf{4 b}$
S-12
g) ${ }^{1} \mathrm{H}$ NMR spectrum of tandem product $\mathbf{4 g}$
h) ${ }^{13} \mathrm{C}\left\{{ }^{1} \mathrm{H}\right\}$ NMR spectrum of $\mathbf{4 g}$
S-14
i) Fractional recrystallization of tandem product $\mathbf{4 b}$
S-15

III. X-ray crystal structures of tandem product $\mathbf{4 b}$ and $\mathbf{4 g}$ 


\section{Experimental Details}

I-a. General Procedures and Materials. Methanol- $d_{4}$, chloroform- $d$ and dichloromethane- $d_{2}$ were purchased from Cambridge Isotope. Aromatic nitrostyrenes were obtained from commercial sources and were used as received. Purification of reaction products was carried out by column chromatography using Silica Gel (40-140 mesh). TLC visualization was accomplished using UV light or with $\mathrm{KMnO}_{4}$ solution. Dichloromethane, dichloroethane, diethyl-ether, hexanes and tetrahydrofuran were purified as described by Grubbs. ${ }^{1}$ All NMR spectra were recorded on either an Avance 400 or Avance $500 \mathrm{MHz}$ spectrometer. ${ }^{1} \mathrm{H}$ and ${ }^{13} \mathrm{C}$ chemical shifts ( $\square$ in ppm) are relative to tetramethylsilane and referenced using chemical shifts of residual solvent resonances. ${ }^{31} \mathrm{P}$ chemical shifts $(\square$ in ppm) are relative to an external $85 \%$ solution of phosphoric acid in the appropriate solvent. Data are reported as $\mathrm{s}$ $=$ singlet, $\mathrm{d}=$ doublet, $\mathrm{t}=$ triplet, $\mathrm{q}=$ quartet, quint $=$ quintet, $\mathrm{m}=$ multiplet, $\mathrm{b}=$ broad, and dd $=$ doublet of doublets with integration and coupling constant(s) given in Hz. Infrared spectra were recorded on 8400S Shimadzu FTIR spectrometer. High-resolution mass spectra were obtained using a ThermoFinnigan MAT95XL high resolution magnetic sector mass spectrometer at the Chemistry Mass Spectrometry Facility at the University of Buffalo. Unless otherwise stated, all reactions and manipulations were performed in dry glassware under a nitrogen atmosphere using either standard Schlenk techniques or an inert-atmosphere glovebox.

-b. Syntheses of the Catalyst, Nazarov substrates and nitrostyrene: The following compounds were made according to literature procedures: $[\operatorname{IrMe}(\mathrm{CO})(\mathrm{dppe})(\mathrm{DIB})]\left(\mathrm{BAr}{ }_{4}\right)_{2},{ }^{2}$ the different Nazarov substrates, ${ }^{3}$ and $n$-propylnitrostyrene. ${ }^{4}$ 
Supporting Info.

\section{General Procedure and Characterization data}

\section{II-a. General Procedure for the $\left[\operatorname{IrMe}(\mathrm{CO})\left(\operatorname{dppe}_{(\mathrm{DIB})}\right)\right]\left(\mathrm{BAr}_{4}\right)_{2}-\mathrm{Catalyzed}$ Tandem}

Nazarov Cyclization-Michael Addition. In a glovebox, a three neck flask equipped with a stir bar and reflux condenser was charged with $[\operatorname{IrMe}(\mathrm{CO})(\mathrm{dppe})(\mathrm{DIB})]\left(\mathrm{BAr}_{4}\right)_{2}(16.4 \mathrm{mg}, 0.006 \mathrm{mmol})$, the Nazarov substrate $(0.15 \mathrm{mmol})$, the nitroalkene Michael acceptor $(0.30 \mathrm{mmol}), N$-ethylpiperidine $(0.015$ mmol) and 1, 2-dichloroethane $(0.75 \mathrm{~mL})$. The reaction solution was stirred at $40{ }^{\circ} \mathrm{C}$ under nitrogen. The volatile components were then removed under reduced pressure and the crude reaction product was purified by column chromatography using ethyl acetate/hexanes (1:1).

\section{II-b. Characterization data for $4 a-4 i$}

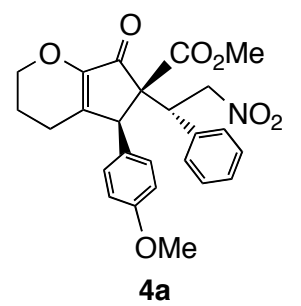

Methyl 5-(4-methoxyphenyl)-6-(2-nitro-1-phenylethyl)-7-oxo-2,3,4,5,6,7-

hexahydropenta[b]pyran-6-carboxylate 4a. ${ }^{1} \mathrm{H} \mathrm{NMR}\left(500 \mathrm{MHz}, \mathrm{CDCl}_{3}\right)$ at $25^{\circ} \mathrm{C}: \square 7.38-7.30(\mathrm{~m}$, $5 \mathrm{H}), 7.06(\mathrm{~d}, J=8.0 \mathrm{~Hz}, 2 \mathrm{H}), 6.93(\mathrm{~d}, J=8.0 \mathrm{~Hz}, 2 \mathrm{H}), 5.31(\mathrm{dd}, J=13.0,11.6 \mathrm{~Hz}, 1 \mathrm{H}), 4.90(\mathrm{dd}, J=$ 13.0, $2.8 \mathrm{~Hz}, 1 \mathrm{H}), 4.51(\mathrm{dd}, J=11.6,2.8 \mathrm{~Hz}, 1 \mathrm{H}), 4.21(\mathrm{~m}, 1 \mathrm{H}), 4.10(\mathrm{~m}, 1 \mathrm{H}), 3.87(\mathrm{~s}, 3 \mathrm{H}), 3.67(\mathrm{~s}, 1 \mathrm{H})$, $3.30(\mathrm{~s}, 3 \mathrm{H}), 2.16(\mathrm{~m}, 1 \mathrm{H}), 1.93(\mathrm{~m}, 1 \mathrm{H}), 1.71(\mathrm{~m}, 2 \mathrm{H}) ;{ }^{13} \mathrm{C}\left\{{ }^{1} \mathrm{H}\right\} \mathrm{NMR}\left(100 \mathrm{MHz}, \mathrm{CDCl}_{3}\right)$ at $25{ }^{\circ} \mathrm{C}: \square$ $196.8,169.4,159.3,152.1,145.9,134.1,130.3,130.3,128.6,128.6,127.1,113.8,77.1,67.1,64.1,55.2$, 52.3, 52.1, 48.3, 22.1, 21.0; IR (cell, $\left.\mathrm{CH}_{2} \mathrm{Cl}_{2}, \mathrm{~cm}^{-1}\right)$ 2950, 2837, 1737, 1710, 1657, 1553, 1512, 1250, 1130, 1037, 841, 798, 706; HRMS (ES $\left.{ }^{+}\right)$: Calculated for $\mathrm{C}_{25} \mathrm{H}_{25} \mathrm{O}_{7} \mathrm{NNa}(\mathrm{M}+\mathrm{Na})^{+} 474.1523$, Found: 474.1538. 


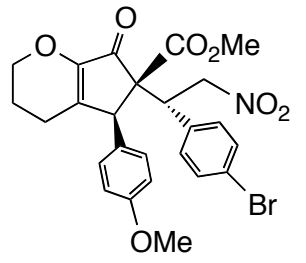

4b
Methyl 5-(4-bromophenyl)-6-(1-(4-methoxyphenyl)-2-nitroethyl)-7-oxo-

2,3,4,5,6,7-hexahydrocyclopenta[b]pyran-6-carboxylate $4 \mathrm{~b} .{ }^{1} \mathrm{H} \mathrm{NMR}\left(400 \mathrm{MHz}, \mathrm{CDCl}_{3}\right)$ at $25^{\circ} \mathrm{C}:[$ $7.47(\mathrm{~d}, J=8.4 \mathrm{~Hz}, 2 \mathrm{H}), 7.18(\mathrm{~d}, J=8.4 \mathrm{~Hz}, 2 \mathrm{H}), 6.99(\mathrm{~d}, J=8.4 \mathrm{~Hz}, 2 \mathrm{H}), 6.86(\mathrm{~d}, J=8.4 \mathrm{~Hz}, 2 \mathrm{H}), 5.25$ $(\mathrm{dd}, J=13.0,11.6 \mathrm{~Hz}, 1 \mathrm{H}), 4.83(\mathrm{dd}, J=13.0,3.2 \mathrm{~Hz}, 1 \mathrm{H}), 4.43(\mathrm{dd}, J=11.6,3.2 \mathrm{~Hz}, 1 \mathrm{H}), 4.12(\mathrm{~m}$, $1 \mathrm{H}), 4.04(\mathrm{~m}, 1 \mathrm{H}), 3.81(\mathrm{~s}, 3 \mathrm{H}), 3.60(\mathrm{~s}, 1 \mathrm{H}), 3.23(\mathrm{~s}, 3 \mathrm{H}), 2.10(\mathrm{~m}, 1 \mathrm{H}), 1.87(\mathrm{~m}, 1 \mathrm{H}), 1.61(\mathrm{~m}, 2 \mathrm{H})$; ${ }^{13} \mathrm{C}\left\{{ }^{1} \mathrm{H}\right\}$ NMR $\left(100 \mathrm{MHz}, \mathrm{CDCl}_{3}\right)$ at $25{ }^{\circ} \mathrm{C}: \square 196.4,169.1,159.4,152.1,145.8,133.4,131.8,131.8$, 130.2, 126.8, 122.7, 113.8, 77.1, 67.1, 64.0, 55.2, 52.3, 52.1, 47.7, 22.2, 21.0; IR (cell, $\left.\mathrm{CH}_{2} \mathrm{Cl}_{2}, \mathrm{~cm}^{-1}\right)$ 2951, 2837, 1736, 1709, 1649, 1553, 1512, 1248, 1130, 1038, 841, 749; HRMS (ES+): Calculated for $\mathrm{C}_{25} \mathrm{H}_{24} \mathrm{O}_{7} \mathrm{NBrNa}(\mathrm{M}+\mathrm{Na})^{+}$554.0608, Found: 554.0607.

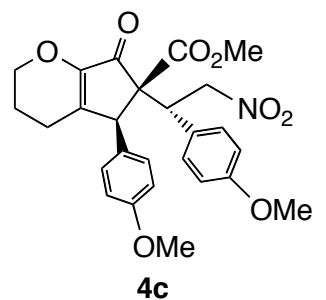

Methyl 5-(4-methoxyphenyl)-6-(1-(4-methoxyphenyl)-2-nitroethyl)-7-oxo2,3,4,5,6,7-hexahydrocyclopenta[b]pyran-6-carboxylate 4c. ${ }^{1} \mathrm{H} \mathrm{NMR}\left(500 \mathrm{MHz}, \mathrm{CDCl}_{3}\right)$ at $25^{\circ} \mathrm{C}:$ $7.20(\mathrm{~d}, J=8.5 \mathrm{~Hz}, 2 \mathrm{H}), 7.00(\mathrm{~d}, J=8.5 \mathrm{~Hz}, 2 \mathrm{H}), 6.86(\mathrm{~d}, J=7.5 \mathrm{~Hz}, 2 \mathrm{H}), 6.83(\mathrm{~d}, J=7.5 \mathrm{~Hz}, 2 \mathrm{H}), 5.24$ $(\mathrm{dd}, J=13.5,11.5 \mathrm{~Hz}, 1 \mathrm{H}), 4.81(\mathrm{dd}, J=13.5,3.0 \mathrm{~Hz}, 1 \mathrm{H}), 4.40(\mathrm{dd}, J=11.5,3.0 \mathrm{~Hz}, 1 \mathrm{H}), 4.13(\mathrm{~m}$, 1H), $4.01(\mathrm{~m}, 1 \mathrm{H}), 3.82(\mathrm{~s}, 3 \mathrm{H}), 3.80(\mathrm{~s}, 3 \mathrm{H}), 3.67(\mathrm{~s}, 1 \mathrm{H}), 3.21(\mathrm{~s}, 3 \mathrm{H}), 2.08(\mathrm{~m}, 1 \mathrm{H}), 1.84(\mathrm{~m}, 1 \mathrm{H}), 1.64$ $(\mathrm{m}, 2 \mathrm{H}) ;{ }^{13} \mathrm{C}\left\{{ }^{1} \mathrm{H}\right\}$ NMR $\left(125 \mathrm{MHz}, \mathrm{CDCl}_{3}\right)$ at $25{ }^{\circ} \mathrm{C}: \square 197.5,170.1,160.2,160.0,152.8,146.5,131.6$, 131.0, 127.9, 126.6, 114.7, 114.4, 78.0, 77.61, 67.7, 65.0, 55.9, 52.8, 52.6, 48.3, 22.8, 21.7; IR (cell, 
$\mathrm{CH}_{2} \mathrm{Cl}_{2}, \mathrm{~cm}^{-1}$ ) 2951, 2837, 1736, 1706, 1649, 1553, 1512, 1250, 1180, 1038, 841; HRMS (ES+):

Calculated for $\mathrm{C}_{26} \mathrm{H}_{27} \mathrm{O}_{8} \mathrm{NNa}(\mathrm{M}+\mathrm{Na})^{+}$504.1629, Found: 504.1618.

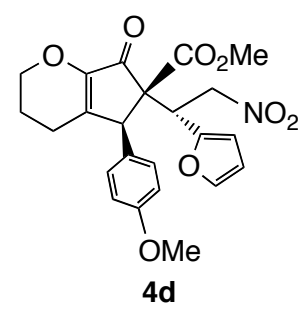

Methyl 6-(1-(furan-2-yl)-2-nitroethyl)-5-(4-methoxyphenyl)-7-oxo-2,3,4,5,6,7hexahydrocyclopenta[b]pyran-6-carboxylate 4 d. ${ }^{1} \mathrm{H}$ NMR $\left(400 \mathrm{MHz}, \mathrm{CDCl}_{3}\right)$ at $25{ }^{\circ} \mathrm{C}: \square 7.36(\mathrm{~d}, \mathrm{~J}$ $=1.6 \mathrm{~Hz}, 1 \mathrm{H}), 7.02(\mathrm{~d}, J=8.8 \mathrm{~Hz}, 2 \mathrm{H}), 6.85(\mathrm{~d}, J=8.8 \mathrm{~Hz}, 2 \mathrm{H}), 6.35(\mathrm{~d}, J=3.2 \mathrm{~Hz}, 1 \mathrm{H}), 6.32(\mathrm{dd}, J=$ 3.2, $1.6 \mathrm{~Hz}, 1 \mathrm{H}), 5.24(\mathrm{dd}, J=14.0,11.2 \mathrm{~Hz}, 1 \mathrm{H}), 4.77(\mathrm{dd}, J=14.0,3.2 \mathrm{~Hz}, 1 \mathrm{H}), 4.62(\mathrm{dd}, J=11.2,3.2$ Hz, 1H), $4.14(\mathrm{~m}, 1 \mathrm{H}), 4.08(\mathrm{~m}, 1 \mathrm{H}), 3.85(\mathrm{~s}, 1 \mathrm{H}), 3.81(\mathrm{~s}, 3 \mathrm{H}), 3.19(\mathrm{~s}, 3 \mathrm{H}), 2.12(\mathrm{~m}, 1 \mathrm{H}), 1.89(\mathrm{~m}, 1 \mathrm{H})$, $1.78(\mathrm{~m}, 2 \mathrm{H}) ;{ }^{13} \mathrm{C}\left\{{ }^{1} \mathrm{H}\right\} \mathrm{NMR}\left(125 \mathrm{MHz}, \mathrm{CDCl}_{3}\right)$ at $25^{\circ} \mathrm{C}: \square 197.5,171.3,162.4,158.2,156.8,142.4$, 131.6, 129.4, 120.9, 116.6, 110.8, 106.4, 75.0, 71.6, 66.7, 56.0, 52.9, 47.2, 31.2, 26.1, 21.7; IR (cell, $\left.\mathrm{CH}_{2} \mathrm{Cl}_{2}, \mathrm{~cm}^{-1}\right)$ 2953, 2837, 1734, 1713, 1651, 1557, 1512, 1252, 1134, 1040, 843, 737; HRMS (ES ${ }^{+}$): Calculated for $\mathrm{C}_{23} \mathrm{H}_{23} \mathrm{O}_{8} \mathrm{NNa}(\mathrm{M}+\mathrm{Na})^{+}$464.1316, Found: 464.1306.

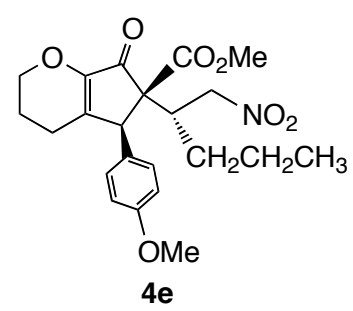

Methyl 5-(4-methoxyphenyl)-6-(1-nitropentan-2-yl)-7-oxo-2,3,4,5,6,7-

hexahydrocyclopenta[b]pyran-6-carboxylate 4 e. ${ }^{1} \mathrm{H} \mathrm{NMR}\left(400 \mathrm{MHz}, \mathrm{CDCl}_{3}\right)$ at $25{ }^{\circ} \mathrm{C}: \mathrm{\square} 7.02(\mathrm{~d}, J$ $=8.4 \mathrm{~Hz}, 2 \mathrm{H}), 6.85(\mathrm{~d}, J=8.4 \mathrm{~Hz}, 2 \mathrm{H}), 4.51(\mathrm{dd}, J=13.0,3.2 \mathrm{~Hz}, 1 \mathrm{H}), 4.42(\mathrm{dd}, J=11.2,3.2 \mathrm{~Hz}, 1 \mathrm{H})$, $4.14(\mathrm{~m}, 1 \mathrm{H}), 4.01(\mathrm{~m}, 1 \mathrm{H}), 3.81(\mathrm{~s}, 3 \mathrm{H}), 3.35(\mathrm{~s}, 1 \mathrm{H}), 3.12(\mathrm{~s}, 3 \mathrm{H}), 2.5(\mathrm{~m}, 1 \mathrm{H}), 2.12(\mathrm{~m}, 1 \mathrm{H}), 1.89(\mathrm{~m}$, 1H), $1.78(\mathrm{~m}, 2 \mathrm{H}$ ), 1.33 (quint, $J=3.5 \mathrm{~Hz}, 2 \mathrm{H}$ ), 1.25 (quint, $J=3.5 \mathrm{~Hz}, 2 \mathrm{H}$ ), $0.96(\mathrm{t}, J=3.5 \mathrm{~Hz}, 3 \mathrm{H}$ ); 
Supporting Info.

${ }^{13} \mathrm{C}\left\{{ }^{1} \mathrm{H}\right\} \mathrm{NMR}\left(125 \mathrm{MHz}, \mathrm{CDCl}_{3}\right)$ at $25{ }^{\circ} \mathrm{C}: \quad \mathrm{1} 194.9,171.2,162.4,157.9,132.2,129.2,119.6,114.1$,

75.1, 71.1, 65.6, 55.9, 52.2, 32.5, 29.8, 26.2, 25.3, 21.7, 19.3, 14.4; IR (cell, $\left.\mathrm{CH}_{2} \mathrm{Cl}_{2}, \mathrm{~cm}^{-1}\right)$ 2956, 2836, 1740, 1713, 1649, 1553, 1512, 1250, 1122, 1034, 837, 733; HRMS (ES+): Calculated for $\mathrm{C}_{22} \mathrm{H}_{27} \mathrm{O}_{7} \mathrm{NNa}(\mathrm{M}+\mathrm{Na})^{+} 440.4529$, Found: 440.4521.

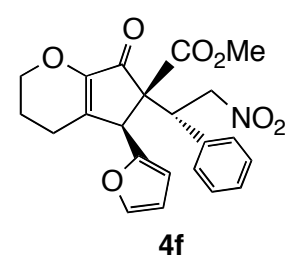

Methyl 5-(furan-2-yl)-6-(2-nitro-1-phenylethyl)-7-oxo-2,3,4,5,6,7hexahydrocyclopenta[b]pyran-6-carboxylate 4 f. ${ }^{1} \mathrm{H} \mathrm{NMR}\left(500 \mathrm{MHz}, \mathrm{CDCl}_{3}\right)$ at $25{ }^{\circ} \mathrm{C}: \square 7.36(\mathrm{~d}, J=$ $1.6 \mathrm{~Hz}, 1 \mathrm{H}), 7.18-7.05(\mathrm{~m}, 5 \mathrm{H}), 6.34(\mathrm{~d}, J=3.2 \mathrm{~Hz}, 1 \mathrm{H}), 6.28(\mathrm{dd}, J=3.2,1.6 \mathrm{~Hz}, 1 \mathrm{H}), 5.23(\mathrm{dd}, J=$ 13.6, 11.2 Hz, 1H), $4.77(\mathrm{dd}, J=13.6,3.2 \mathrm{~Hz}, 1 \mathrm{H}), 4.60(\mathrm{dd}, J=11.2,3.2 \mathrm{~Hz}, 1 \mathrm{H}), 4.13(\mathrm{~m}, 1 \mathrm{H}), 4.08$ $(\mathrm{m}, 1 \mathrm{H}), 3.85(\mathrm{~s}, 1 \mathrm{H}), 3.17(\mathrm{~s}, 3 \mathrm{H}), 2.11(\mathrm{~m}, 1 \mathrm{H}), 1.86(\mathrm{~m}, 1 \mathrm{H}), 1.78(\mathrm{~m}, 2 \mathrm{H}) ;{ }^{13} \mathrm{C}\left\{{ }^{1} \mathrm{H}\right\} \mathrm{NMR}(125$ $\left.\mathrm{MHz}, \mathrm{CDCl}_{3}\right)$ at $25^{\circ} \mathrm{C}: \square 195.3,169.6,152.9,150.4,143.3,142.7,142.2,134.4,128.6,111.0 .109 .6$, 109.2, 77.6, 67.1, 56.3, 52.7, 48.0, 46.8, 22.1, 21.2; IR (cell, $\left.\mathrm{CH}_{2} \mathrm{Cl}_{2}, \mathrm{~cm}^{-1}\right)$ 2953, 2836, 1736, 1713, 1653, 1553, 1435,1379, 1238, 737, 704; HRMS $\left(\mathrm{ES}^{+}\right)$: Calculated for $\mathrm{C}_{22} \mathrm{H}_{21} \mathrm{O}_{7} \mathrm{NNa}(\mathrm{M}+\mathrm{Na})^{+}$ 434.1210, Found: 434.1199.

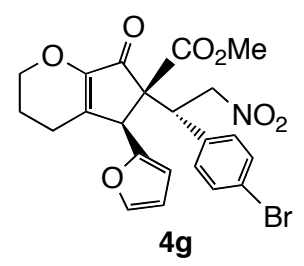

Methyl 6-(1-(4-bromophenyl)-2-nitroethyl)-5-(furan-2-yl)-7-oxo-2,3,4,5,6,7hexahydrocyclopenta[b]pyran-6-carboxylate 4 g. ${ }^{1} \mathrm{H}$ NMR $\left(500 \mathrm{MHz}, \mathrm{CDCl}_{3}\right)$ at $25{ }^{\circ} \mathrm{C}: \square 7.46(\mathrm{~d}, J$ $=8.5 \mathrm{~Hz}, 2 \mathrm{H}), 7.40(\mathrm{~d}, J=1.2 \mathrm{~Hz}, 1 \mathrm{H}), 7.17(\mathrm{~d}, J=8.5 \mathrm{~Hz}, 2 \mathrm{H}), 6.35(\mathrm{dd}, J=1.2,3.0 \mathrm{~Hz}, 1 \mathrm{H}), 6.10(\mathrm{~d}$, 
Supporting Info.

$J=1.0 \mathrm{~Hz}, 1 \mathrm{H}), 5.30(\mathrm{dd}, J=14,11.5 \mathrm{~Hz}, 1 \mathrm{H}), 4.86(\mathrm{dd}, J=14,3.0 \mathrm{~Hz}, 1 \mathrm{H}), 4.46(\mathrm{dd}, J=11.5,3.0 \mathrm{~Hz}$, $1 \mathrm{H}), 4.15(\mathrm{~m}, 1 \mathrm{H}), 4.06(\mathrm{~m}, 1 \mathrm{H}), 3.81(\mathrm{~s}, 1 \mathrm{H}), 3.46(\mathrm{~s}, 3 \mathrm{H}), 2.20(\mathrm{~m}, 1 \mathrm{H}), 1.90(\mathrm{~m}, 1 \mathrm{H}), 1.76(\mathrm{~m}, 2 \mathrm{H})$; ${ }^{13} \mathrm{C}\left\{{ }^{1} \mathrm{H}\right\}$ NMR $\left(125 \mathrm{MHz}, \mathrm{CDCl}_{3}\right)$ at $25{ }^{\circ} \mathrm{C}: \quad \square 195.3,169.6,152.9,150.4,143.3,142.2,134.4,132.5$, 131.9, 123.2, 111.0, 109.2, 77.5, 76.6, 67.1, 52.7, 48.1, 46.7, 22.6, 21.2; IR (cell, $\left.\mathrm{CH}_{2} \mathrm{Cl}_{2}, \mathrm{~cm}^{-1}\right)$ 2961, 2839, 1738, 1713, 1651, 1553, 1514, 1300, 1225, 1183, 1038, 802, 741; HRMS (ES+): Calculated for $\mathrm{C}_{22} \mathrm{H}_{20} \mathrm{O}_{7} \mathrm{NBrNa}(\mathrm{M}+\mathrm{Na})^{+}$512.0315, Found: 512.0316 .

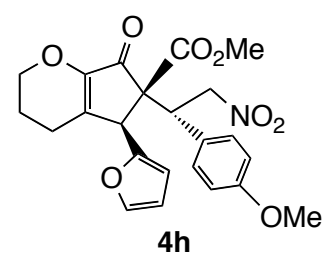

Methyl 5-(furan-2-yl)-6-(1-(4-methoxyphenyl)-2-nitroethyl)-7-oxo-2,3,4,5,6,7-

hexahydrocyclopenta[b]pyran-6-carboxylate $4 \mathbf{h} .{ }^{1} \mathrm{H} \mathrm{NMR}\left(400 \mathrm{MHz}, \mathrm{CDCl}_{3}\right)$ at $25{ }^{\circ} \mathrm{C}: \square 7.49(\mathrm{~d}, J$ $=1.2 \mathrm{~Hz}, 1 \mathrm{H}), 7.29(\mathrm{~d}, J=8.8 \mathrm{~Hz}, 2 \mathrm{H}), 6.92(\mathrm{~d}, J=8.8 \mathrm{~Hz}, 2 \mathrm{H}), 6.44(\mathrm{dd}, J=1.2,3.2 \mathrm{~Hz}, 1 \mathrm{H}), 6.19(\mathrm{~d}$, $J=1.2 \mathrm{~Hz}, 1 \mathrm{H}), 5.38(\mathrm{dd}, J=13.6,11.6 \mathrm{~Hz}, 1 \mathrm{H}), 4.93(\mathrm{dd}, J=13.6,3.2 \mathrm{~Hz}, 1 \mathrm{H}), 4.52(\mathrm{dd}, J=11.6,3.2$ Hz, 1H), $4.22(\mathrm{~m}, 1 \mathrm{H}), 4.12(\mathrm{~m}, 1 \mathrm{H}), 3.97(\mathrm{~s}, 1 \mathrm{H}), 3.87(\mathrm{~s}, 3 \mathrm{H}), 3.54(\mathrm{~s}, 3 \mathrm{H}), 2.27(\mathrm{~m}, 1 \mathrm{H}), 1.99(\mathrm{~m}, 1 \mathrm{H})$, $1.82(\mathrm{~m}, 2 \mathrm{H}) ;{ }^{13} \mathrm{C}\left\{{ }^{1} \mathrm{H}\right\} \mathrm{NMR}\left(125 \mathrm{MHz}, \mathrm{CDCl}_{3}\right)$ at $25^{\circ} \mathrm{C}: \square 196.6,170.0,160.2,152.5,150.1,143.6$, 143.5, 131.4, 126.5, 114.7, 111.2, 109.7, 77.6, 67.7, 63.9, 55.8, 53.4, 47.7, 46.9, 22.7, 21.6; IR (cell, $\left.\mathrm{CH}_{2} \mathrm{Cl}_{2}, \mathrm{~cm}^{-1}\right)$ 2961, 2839, 1738, 1713, 1651, 1553, 1514, 1300, 1225, 1183, 1038, 802, 741; HRMS $\left(\mathrm{ES}^{+}\right)$: Calculated for $\mathrm{C}_{23} \mathrm{H}_{23} \mathrm{O}_{8} \mathrm{NNa}(\mathrm{M}+\mathrm{Na})^{+} 464.4310$, Found: 464.4299 . 
Supporting Info.

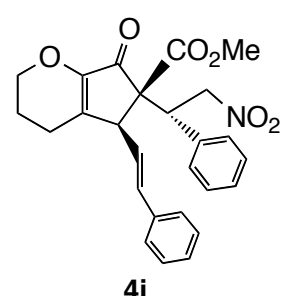

Methyl 6-(2-nitro-1-phenylethyl)-7-oxo-5-styryl-2,3,4,5,6,7-

hexahydrocyclopenta[b]pyran-6-carboxylate $4 \mathbf{i} .{ }^{1} \mathrm{H} \mathrm{NMR}\left(400 \mathrm{MHz}, \mathrm{CDCl}_{3}\right)$ at $25^{\circ} \mathrm{C}: \square 7.27-7.37$ $(\mathrm{m}, 10 \mathrm{H}), 6.59(\mathrm{~d}, J=16 \mathrm{~Hz}, 1 \mathrm{H}), 5.88(\mathrm{dd}, J=6.4,9.2 \mathrm{~Hz}, 1 \mathrm{H}), 5.31(\mathrm{dd}, J=2.4,13.6 \mathrm{~Hz}, 1 \mathrm{H}), 5.01$ $(\mathrm{dd}, J=2.4,13.6 \mathrm{~Hz}, 1 \mathrm{H}), 4.47(\mathrm{dd}, J=2.4,11.2 \mathrm{~Hz}, 1 \mathrm{H}), 4.12(\mathrm{~m}, 1 \mathrm{H}), 3.98(\mathrm{~m}, 1 \mathrm{H}), 3.73(\mathrm{~s}, 3 \mathrm{H})$, $3.28(\mathrm{~d}, J=9.2 \mathrm{~Hz}, 1 \mathrm{H}), 2.17(\mathrm{~m}, 1 \mathrm{H}), 1.85(\mathrm{~m}, 1 \mathrm{H}), 1.62(\mathrm{~m}, 2 \mathrm{H}){ }^{13} \mathrm{C}\left\{{ }^{1} \mathrm{H}\right\} \mathrm{NMR}\left(125 \mathrm{MHz}, \mathrm{CDCl}_{3}\right)$ at $25{ }^{\circ} \mathrm{C}: \square 196.2,169.8,151.2,146.4,136.0,135.9,134.2,129.5,128.7,128.6,128.5,128.2,126.3$, 123.8, 77.1, 67.0, 62.9, 52.7, 50.3, 47.8, 22.3, 21.2; IR (cell, $\left.\mathrm{CH}_{2} \mathrm{Cl}_{2}, \mathrm{~cm}^{-1}\right)$ 2956, 2833, 1733, 1710, 1655, 1550, 1510, 1250, 1233, 1118, 1005, 841, 798, 700 ; HRMS (ES+): Calculated for $\mathrm{C}_{26} \mathrm{H}_{25} \mathrm{O}_{6} \mathrm{NNa}^{+}$ $(\mathrm{M}+\mathrm{Na})^{+} 470.1574$, Found: 470.1579. 
Supporting Info.

II-c. ${ }^{1} \mathrm{H}$ NMR spectrum of methyl 5-(4-methoxyphenyl)-6-(2-nitro-1-phenylethyl)-7-oxo-2,3,4,5,6,7hexahydrocyclopenta[b]pyran-6-carboxylate (4a).
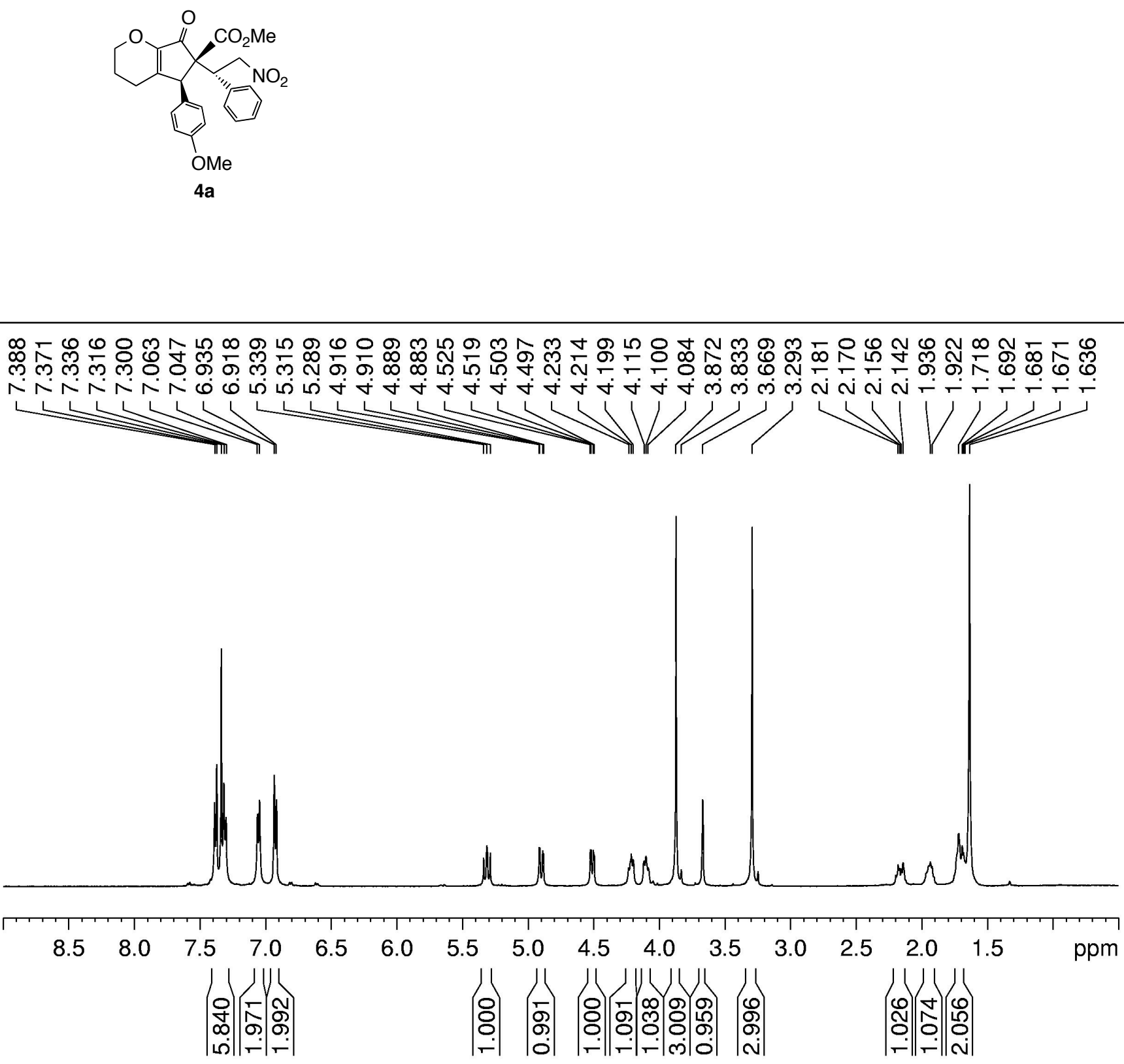
II-d. ${ }^{13} \mathrm{C}\left\{{ }^{1} \mathrm{H}\right\}$ (a), ${ }^{13} \mathrm{C} / \mathrm{DEPT} 135$ (b) and ${ }^{13} \mathrm{C} / \mathrm{DEPT} 90$ (c) NMR spectra of methyl 5-(4-methoxyphenyl)6-(2-nitro-1-phenylethyl)-7-oxo-2,3,4,5,6,7-hexahydrocyclopenta[b]pyran-6-carboxylate (4a).

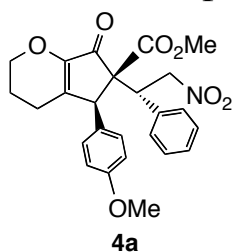

(a)

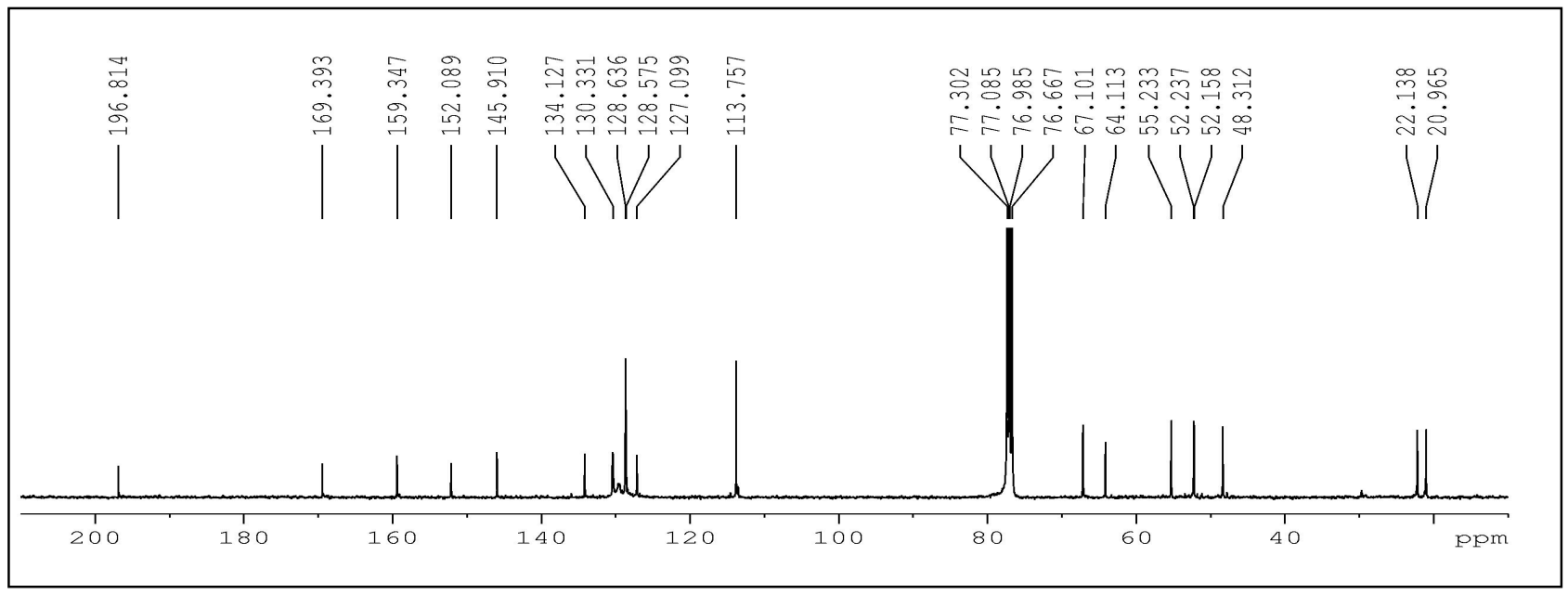

(b)

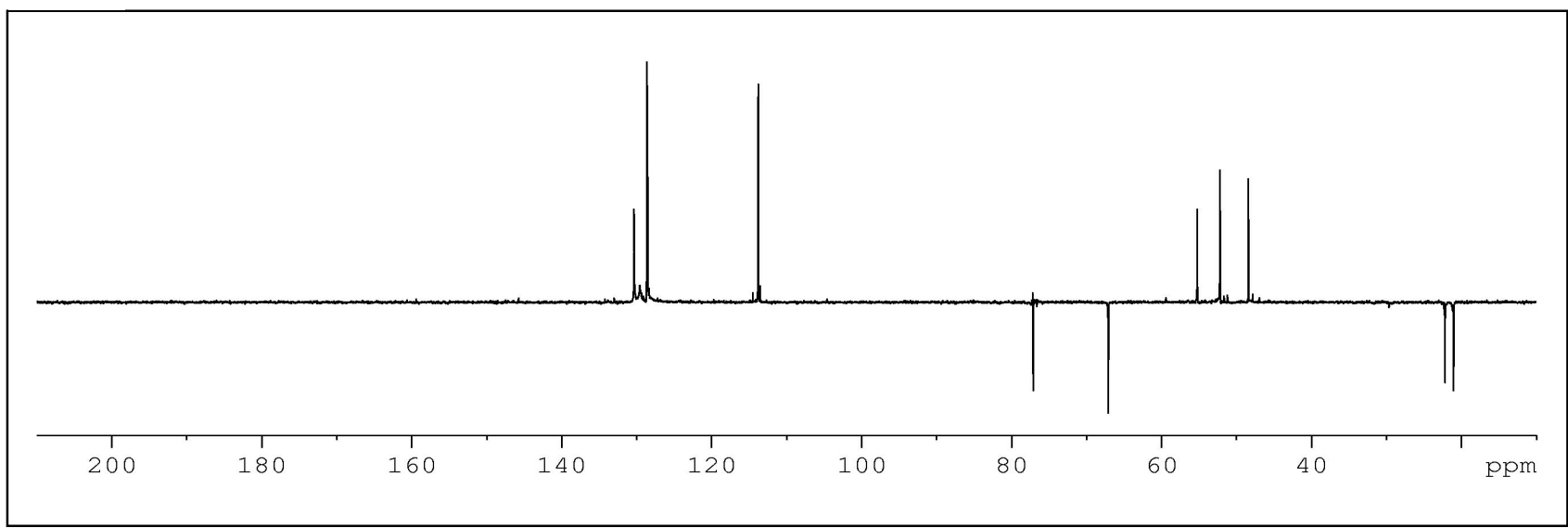

(c)

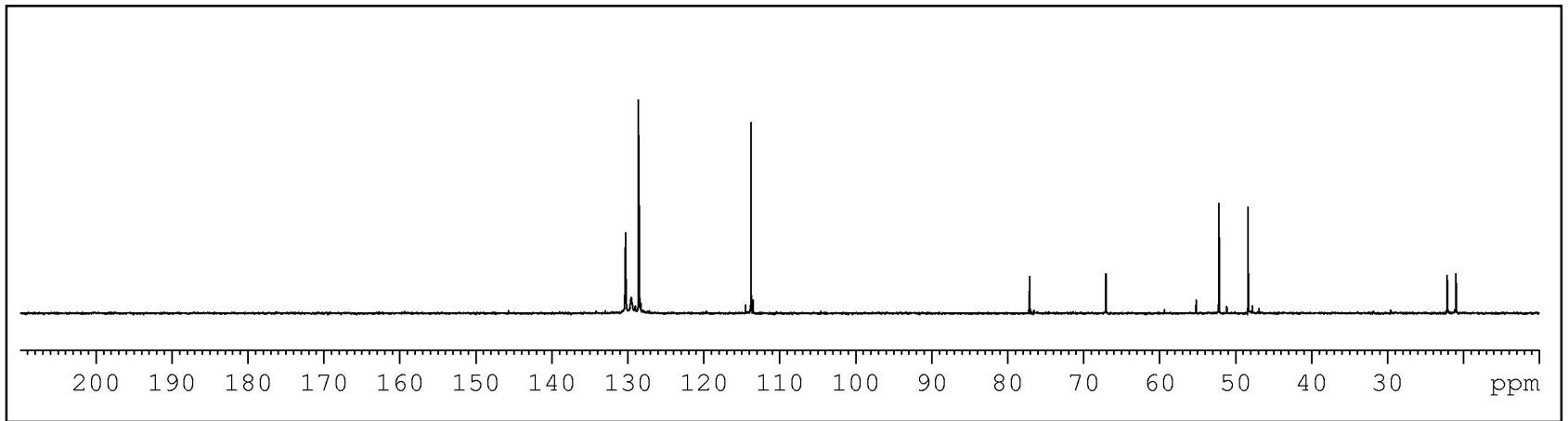


Supporting Info.

II-e. ${ }^{1} \mathrm{H}$ NMR spectrum of methyl 6-(1-(4-bromophenyl)-2-nitroethyl)-5-(4-methoxyphenyl)-7-oxo2,3,4,5,6,7-hexahydrocyclopenta[b]pyran-6-carboxylate (4b).
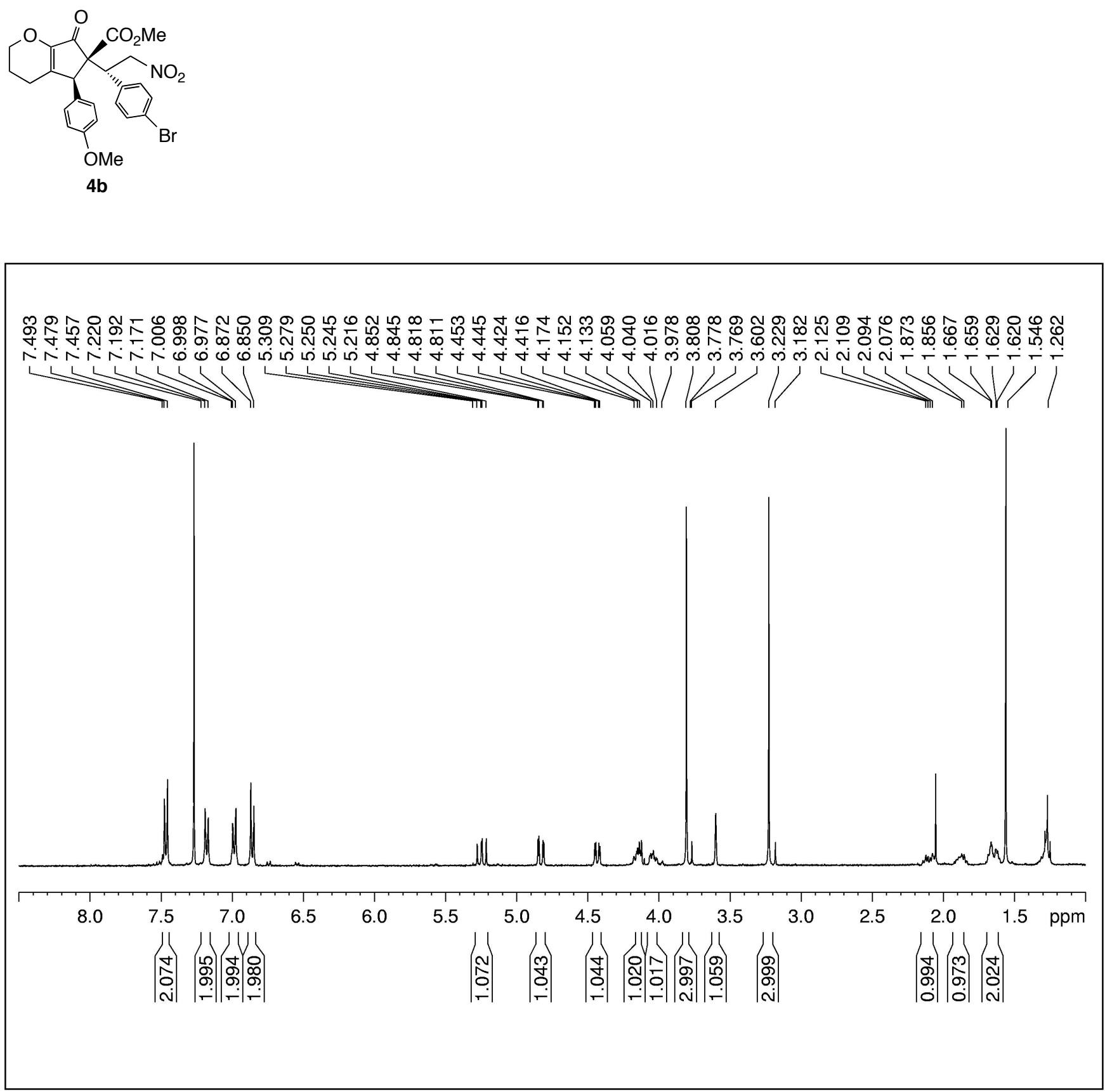
Supporting Info.

II-f. ${ }^{13} \mathrm{C}\left\{{ }^{1} \mathrm{H}\right\}$ (a), ${ }^{13} \mathrm{C} / \mathrm{DEPT} 135$ (b) and ${ }^{13} \mathrm{C} / \mathrm{DEPT} 90$ (c) NMR spectra of methyl 6-(1-(4bromophenyl)-2-nitroethyl)-5-(4-methoxyphenyl)-7-oxo-2,3,4,5,6,7-hexahydrocyclopenta[b]pyran-6carboxylate (4b).

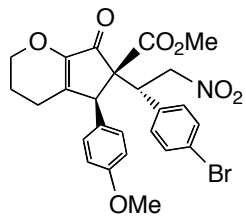

$(\boldsymbol{a})$

4b

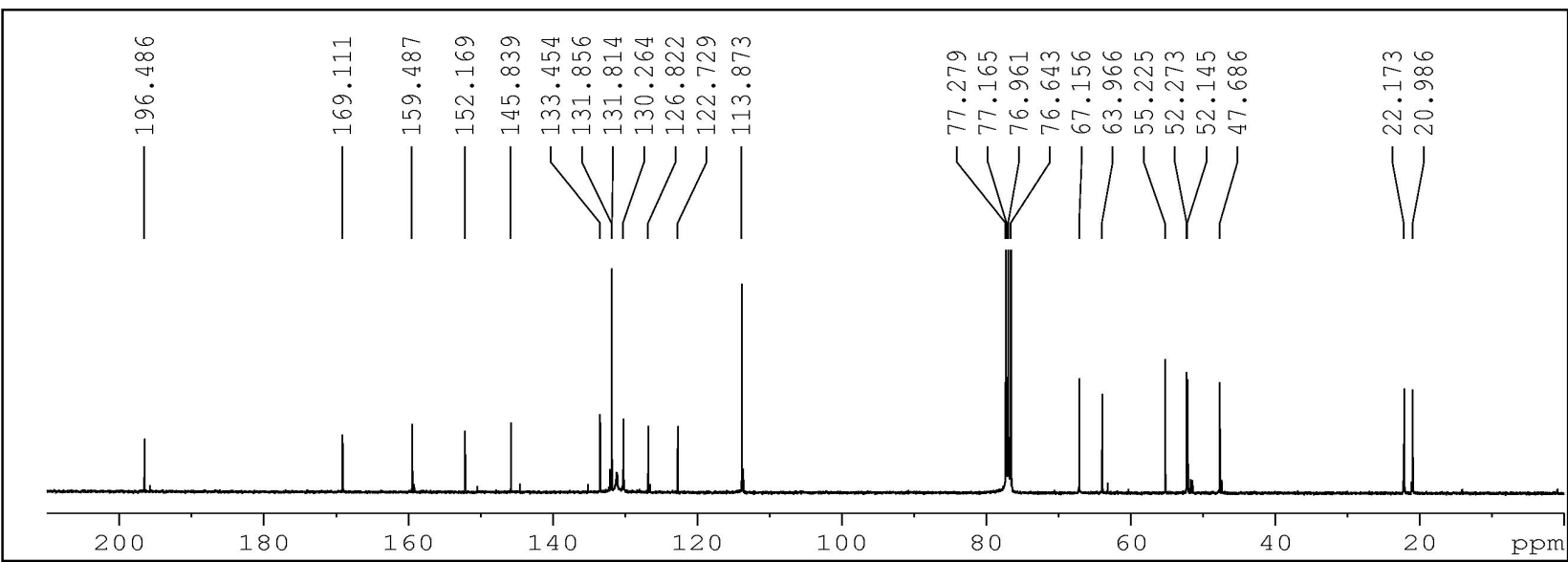

(b)

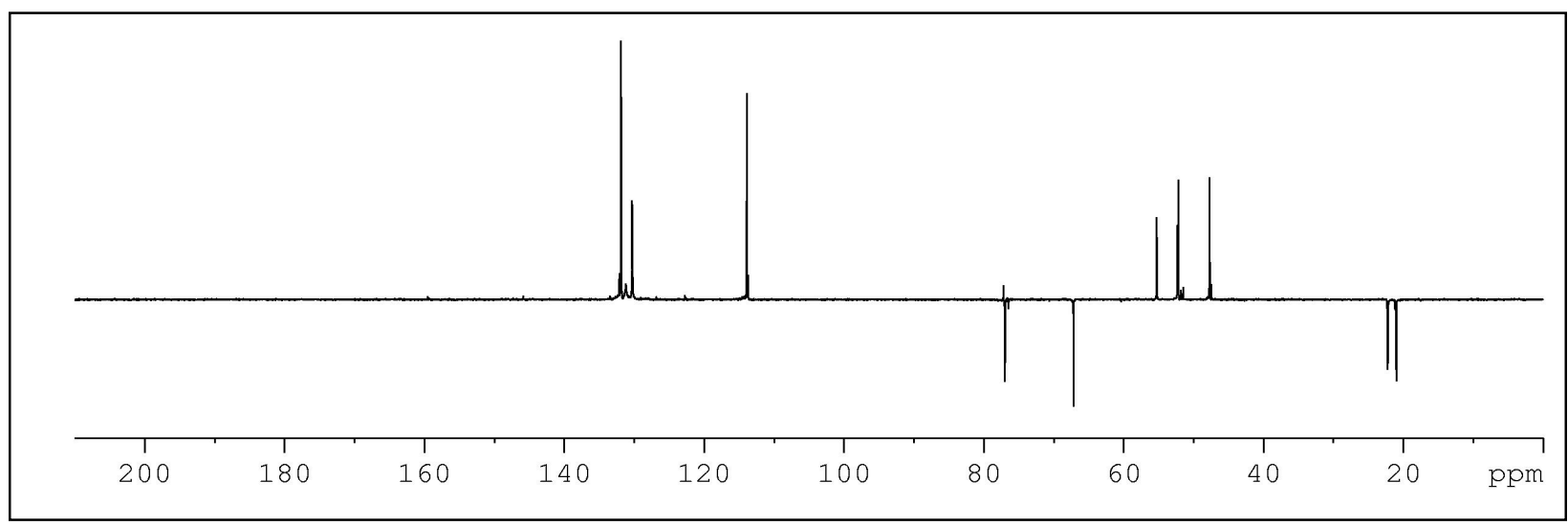

(c)

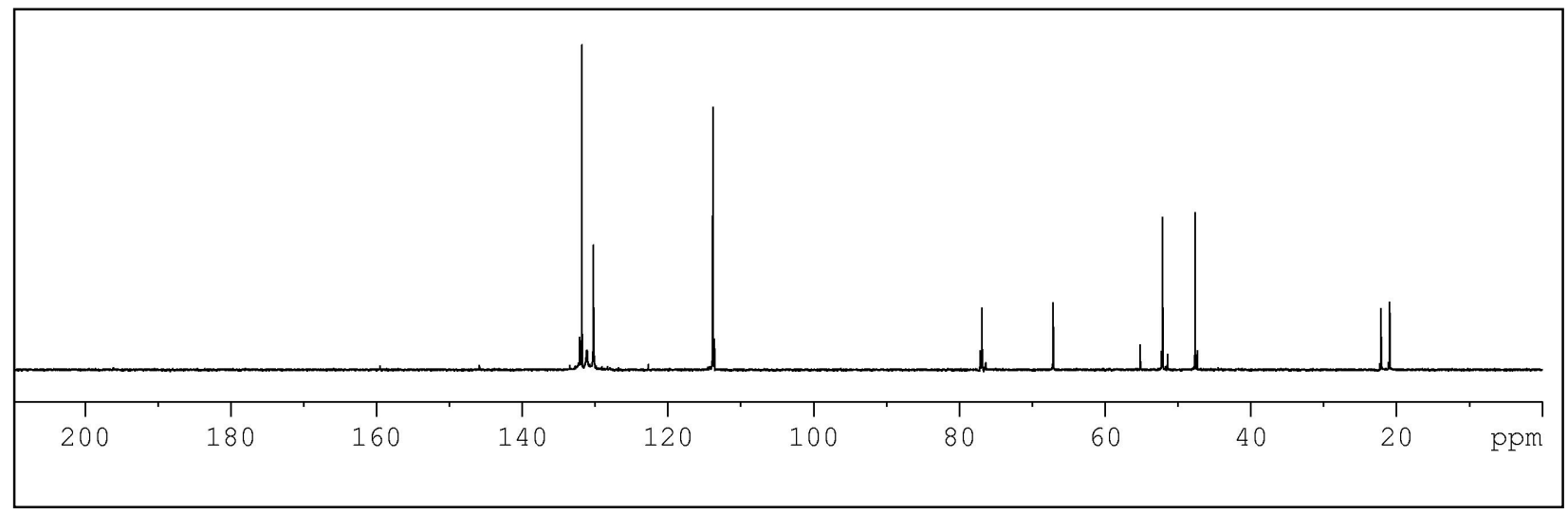


Supporting Info.

II-g. ${ }^{1} \mathrm{H}$ NMR spectrum of methyl 6-(1-(4-bromophenyl)-2-nitroethyl)-5-(furan-2-yl)-7-oxo-2,3,4,5,6,7hexahydrocyclopenta[b]pyran-6-carboxylate (4g).

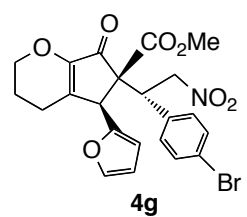

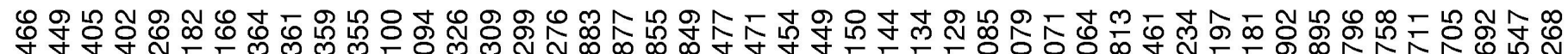

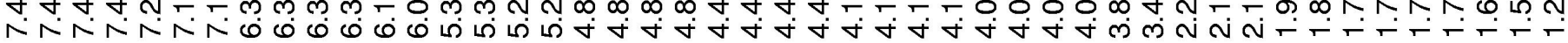
(1)

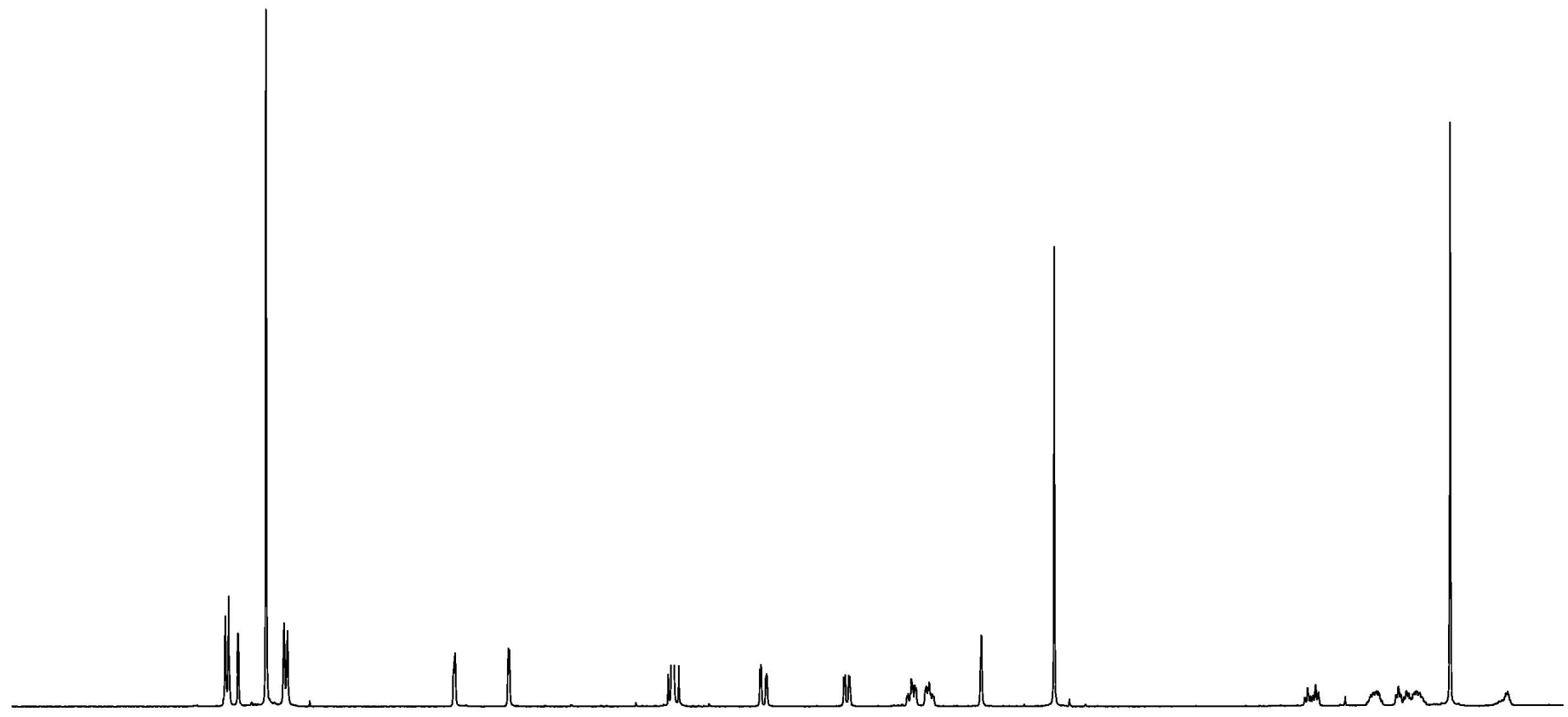

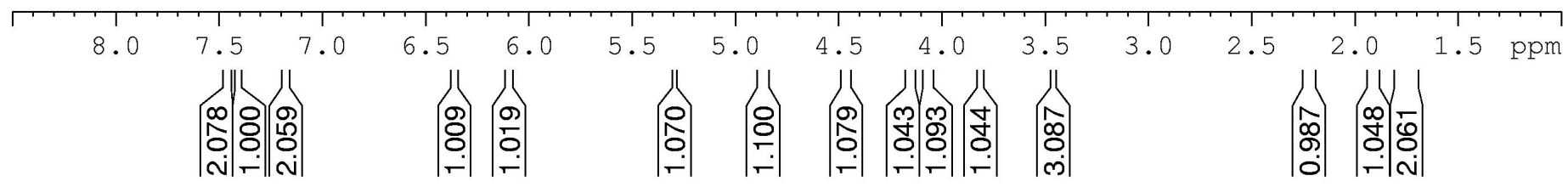


Supporting Info.

II-h. ${ }^{13} \mathrm{C}\left\{{ }^{1} \mathrm{H}\right\}$ NMR spectrum of methyl 6-(1-(4-bromophenyl)-2-nitroethyl)-5-(furan-2-yl)-7-oxo2,3,4,5,6,7-hexahydrocyclopenta[b]pyran-6-carboxylate (4g).

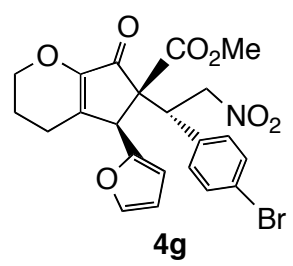

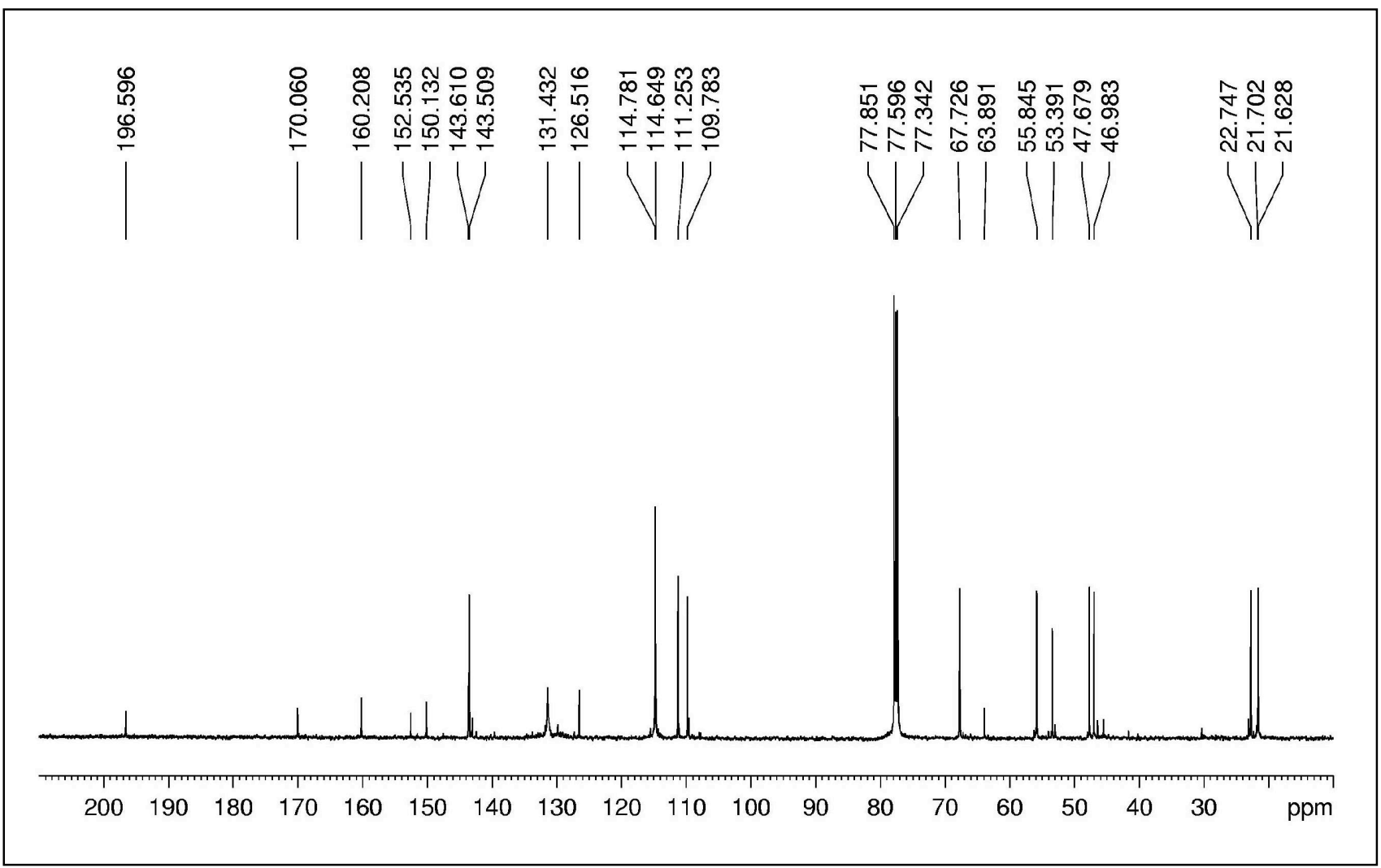


Janka et. al. Tandem Nazarov-Michael

S-15

Supporting Info.

II-i. Fractional recrystallization of tandem product $4 \mathrm{~b}$ :

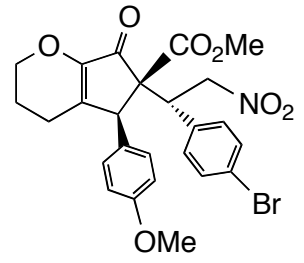

4b

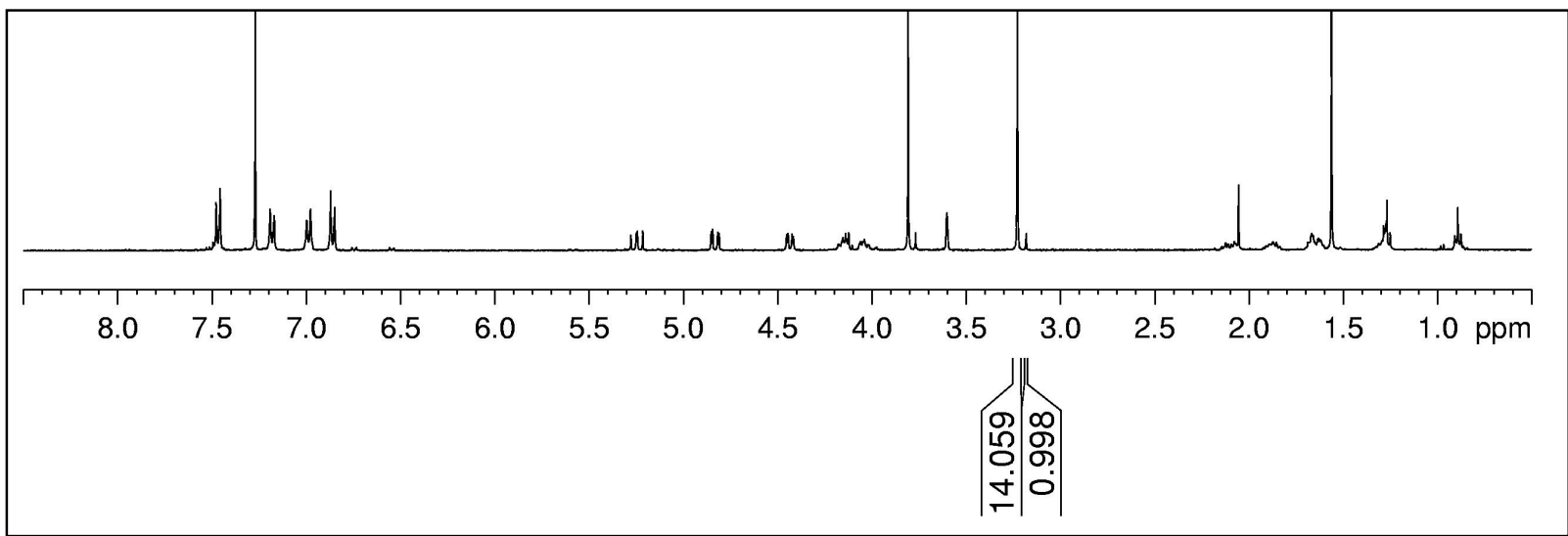

${ }^{1} \mathrm{H}$ NMR spectrum in $\mathrm{CDCl}_{3}$ of product $\mathbf{4 b}$.

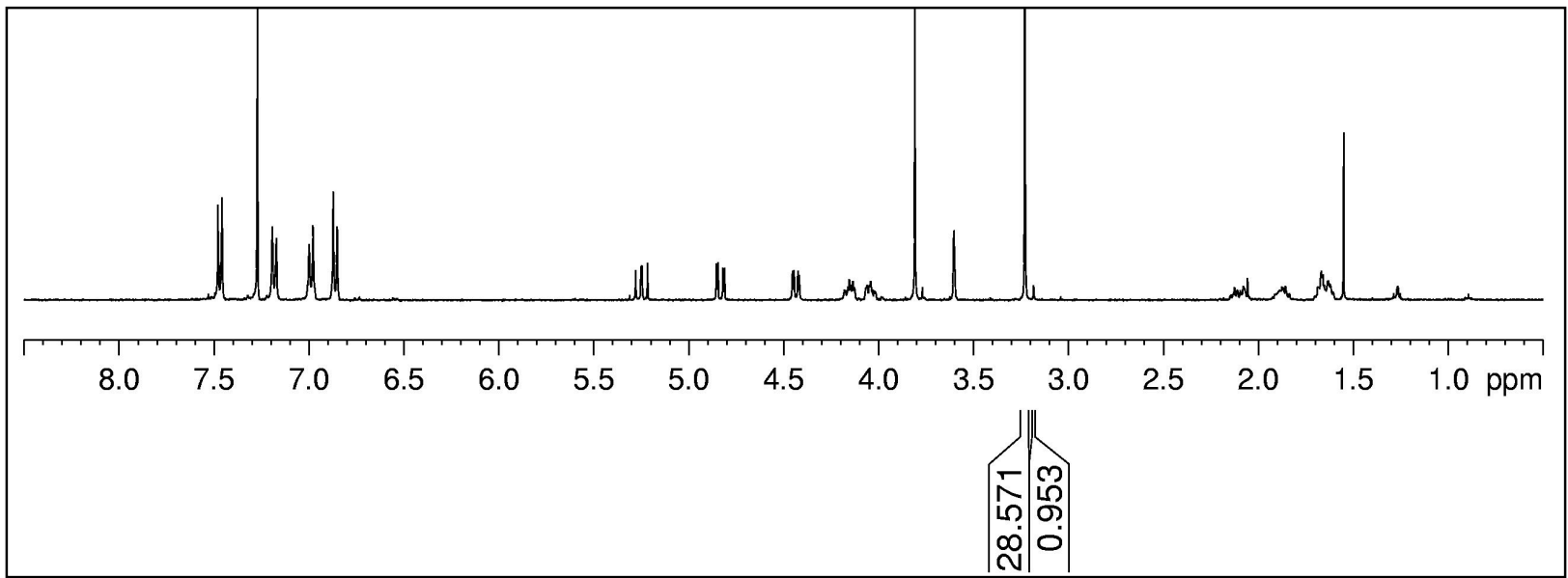

${ }^{1} \mathrm{H}$ NMR spectrum in $\mathrm{CDCl}_{3}$ of the white solid precipitated out after dissolving oily product $\mathbf{4} \mathbf{b}$ in ethyl acetate/hexanes (2:3).

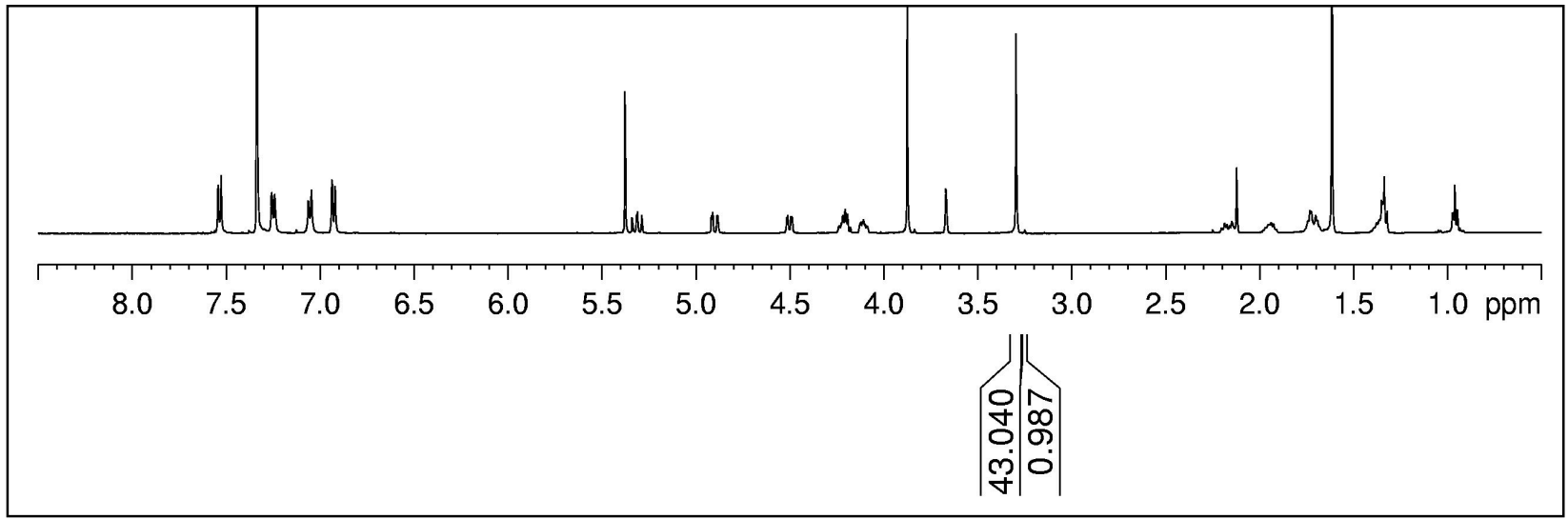

${ }^{1} \mathrm{H}$ NMR spectrum in $\mathrm{CDCl}_{3}$ of the white solid obtained after second fractional recrystallization from ethyl acetate/hexanes (2:3). 
Supporting Info.

\section{X-Ray Crystal Structures of product $4 \mathrm{~b}$ and $4 \mathrm{~g}$}

1. An ORTEP32/pov-ray representation of $\mathbf{4 b}$ showing $50 \%$ probability ellipsoids. For clarity selected $\mathrm{H}$ atoms are shown.
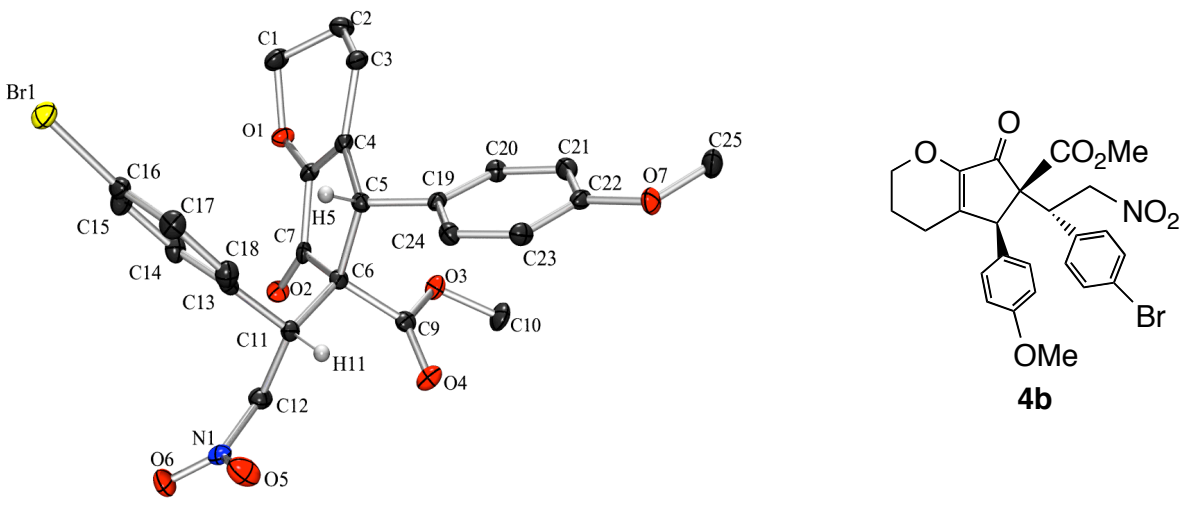

2. An ORTEP32/pov-ray representation of $\mathbf{4 g}$ showing $50 \%$ probability ellipsoids. For clarity selected $\mathrm{H}$ atoms are shown.
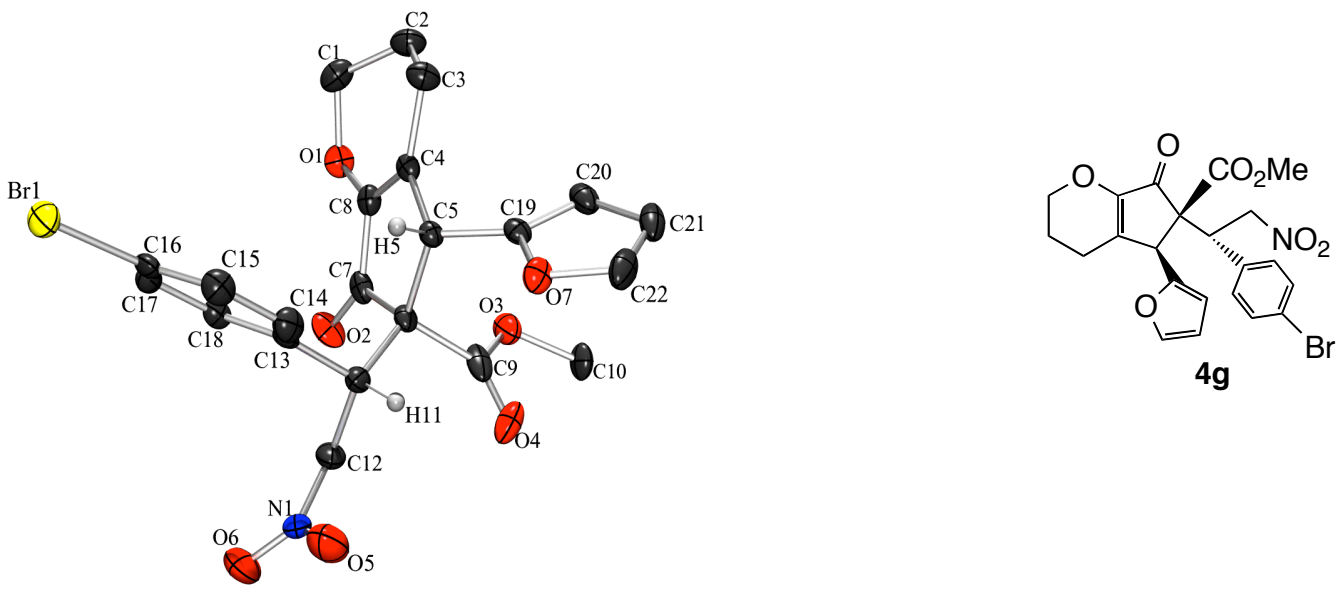
Supporting Info.

\section{References for supporting information:}

(1) Pangborn, A. B.; Giardello, M. A.; Grubbs, R. H.; Rosen, R. K.; Timmers, F. J. Organometallics 1996, 15, 1518-1520.

(2) Albietz, P. J.; Cleary, B. P.; Paw, W.; Eisenberg, R. Inorg. Chem. 2002, 41, 2095-2108.

(3) He, W.; Sun, X.; Frontier, A. J. J. Am. Chem. Soc. 2003, 125, 14278-14279.

(4) Lucet, D.; Sabelle, S.; Kostelitz, O; Gall, T. L.; Mioskowski, C. Eur. J. Org. Chem. 1999, 2583-2581. 